\title{
COVID-19 Is Not the Flu: Four Graphs From Four Countries
}

\begin{abstract}
Jovana Stojanovic ${ }^{1,2 t}$, Vincent G. Boucher ${ }^{1,3+}$, Jacqueline Boyle ${ }^{4,5}$, Joanne Enticott ${ }^{4,6}$, Kim L. Lavoie ${ }^{1,3}$ and Simon L. Bacon ${ }^{1,2 *}$

${ }^{1}$ Montreal Behavioral Medicine Centre, Centre integrée universitaire de santé et services sociaux du Nord de l'lle de Montréal (CIUSSS-NIM), Montreal, QC, Canada, ${ }^{2}$ Department of Health, Kinesiology, and Applied Physiology, Concordia University, Montreal, QC, Canada, ${ }^{3}$ Department of Psychology, University of Quebec at Montreal, Montreal, QC, Canada, ${ }^{4}$ Monash Centre for Health Research and Implementation, Monash University, Clayton, VIC, Australia, ${ }^{5}$ School of Public Health and Preventive Medicine, Monash University, Melbourne, VIC, Australia, ${ }^{6}$ Monash Partners Academic Health Science Centre, Clayton, VIC, Australia
\end{abstract}

Background: COVID-19 has caused a global public health emergency. Government mitigation strategies included a series of behavior-based prevention policies that had a likely impact on the spread of other contagious respiratory illnesses, such as seasonal influenza. Our aim was to explore how 2019-2020 influenza tracked onto COVID-19 pandemic and its mitigation methods.

Materials and Methods: We linked the WHO FluNet database and COVID-19 confirmed cases (Johns Hopkins University) for four countries across the northern (Canada, the United States) and southern hemispheres (Australia, Brazil) for the period 2016-2020. Graphical presentations of longitudinal data were provided.

Results: There was a notable reduction in influenza cases for the 2019-2020 season. Northern hemisphere countries experienced a quicker ending to the 2019-2020 seasonal influenza cases (shortened by 4-7 weeks) and virtually no 2020 fall influenza season. Countries from the southern hemisphere experienced drastically low levels of seasonal influenza, with consistent trends that were approaching zero cases after the introduction of COVID-19 measures.

Conclusions: It is likely that the COVID-19 mitigation measures played a notable role in the marked decrease in influenza, with little to no influenza activity in both the northern and southern hemispheres. In spite of this reduction in influenza cases, there was still community spread of COVID-19, highlighting the contagiousness of SARS-CoV-2 compared to influenza. These results, together with the higher mortality rate from SARS-CoV-2 compared to influenza, highlight that COVID-19 is a far greater health threat than influenza.

Keywords: COVID-19, influenza, transmission, epidemiology, behavior-based policy, behavior change

\section{INTRODUCTION}

On March 11th, 2020, the World Health Organization (WHO) declared COVID-19 a pandemic, a disease caused by the SARS-CoV-2 virus (1). As of December 31st, 2020, there have been around 83.52 million cases in 188 countries, areas, or territories, with a death toll of $\sim 1.82$ million individuals (2). COVID-19 prevention measures have relied upon widespread adherence to 
behavior-based policies, like physical distancing, mask wearing, and hand washing, to reduce virus transmission, even with the current introduction of vaccines in numerous countries (3-5). Theoretically, these mitigation measures should also have positive impacts on other transmissible infectious diseases such as the influenza virus.

Seasonal influenza A and B epidemics generally occur between November and April across the northern hemisphere, and between late May and October across the southern hemisphere (6), time periods which have overlapped with various COVID19 waves in the countries in both hemispheres. As such, we aimed to explore how the epidemiological pattern of the 2019-2020 influenza tracked onto the evolution of the COVID-19 pandemic and the first introduction of behaviorbased mitigation methods to prevent its transmission in four countries (Australia, Brazil, Canada, and the United States). Another aim was to provide evidence to combat the missinformation being spread (7) that the impact of COVID-19 is no different to influenza. These have propagated messages within some communities that adherence to the behaviorbased prevention policies are unnecessary resulting in nonadherence and in some areas, public protesting against the policies (8).

\section{MATERIALS AND METHODS}

\section{Data Sources}

We accessed FluNet data on influenza virological surveillance coordinated and provided by the WHO, between January 1st, 2016, and December 27th, 2020 (9). Influenza number of cases (A [H1]; A [H1N1] pdm09; A[H3]; A[H5]; A[not subtyped]; B [Yamagata lineage]; B [Victoria lineage]; B [lineage not determined]) and total number of influenza positive/negative viruses for Australia, Brazil, Canada, and the United States were downloaded for the period 20162020. Most recent COVID-19 epidemiological data, between January 1st and December 25th, 2020, were obtained from the Johns Hopkins Coronavirus Resources Center $(2,10)$. In order to obtain data on policies related to COVID-19, the Coronavirus Government Response Tracker was used to obtain the dates when the governments had first put in place closures and containment measures (school closing, workplace closing, international travel controls, canceling of public events) (11). For the northern hemisphere, the first containment measures included international travel controls on January 22nd and February 22nd for Canada and the United States, respectively. For the southern hemisphere, the international travel controls in Australia (February 2nd) and the school and workplace closing in Brazil (March 12th) were first introduced. Influenza and COVID-19 datasets were merged based on the weeks of the year (i.e., week $1=$ the first week in January), and graphical presentations of the raw longitudinal data are provided in order to obtain instantaneous visual insight and discuss the potential influence of COVID-19 outbreak on influenza rates across the individual countries.

\section{RESULT}

\section{Northern Hemisphere-Examples of Canada and the United States}

Between 2016-2019, the average influenza season occurred between October (week 40) and May (week 19) for both Canada and the United States, with peaks around weeks 7 and 8 (end of February). As seen in Figure 1, there was a significant reduction in influenza cases during the first months of 2020 (solid blue curve) compared to the average number of influenza cases (dashed dark blue curve) after the COVID-19 mitigation measures were introduced (solid black line). Furthermore, this notable decrease in influenza cases (lower than 500 cases/week) meant that the influenza season seemed to end 7 and 4 weeks earlier for the United States and Canada, respectively (week 15; between April 6 and 12, 2020) compared to previous years. In contrast, the number of cases of COVID-19 in both countries (solid red curve) started to increase dramatically during week 11 of 2020 (March 9-15, 2020), while there was a more rapid than usual decline in the number of influenza cases between weeks 12 and 13 (March 16-29, 2020). Finally, we observed persistently low numbers of influenza cases, approaching zero values, in both countries throughout the start of the 2020-2021 influenza season, which contrasts the consistent increases in cases and the second wave of COVID-19 (see Figures 1A,B).

\section{Southern Hemisphere- Examples of Australia and Brazil}

In the southern hemisphere, the average influenza season runs from May (week 19) to November (week 45) in Australia and from March (week 9) to August (week 31) in Brazil, with peaks occurring around week 34 and week 14, respectively (see Figure 2). This pattern is notably different from the northern hemisphere. In Australia, the influenza case rates remained around zero from around week 16 up to when we stopped capturing data (December 27, 2020; week 40). More notable, is that the usual peak in influenza cases (around week 34) did not occur in 2020, this is in spite of there being a 2nd COVID-19 wave which covered this period (weeks 24-40, peak at week 31). In Brazil, the initial phases of the 2020 influenza season followed a normal pattern until week 12 ( 1 week after the introduction of the COVID-19 mitigation measures of the 4 countries included). By week 16 (mid-April, 2020) there were minimal influenza cases and by week 19 (early May, 2020) the case rate dropped to zero, where it remained until the end of data capture (December 27, 2020; week 40). In contrast, the number of COVID-19 cases started to increase around week 13, and have remained elevated ever since.

\section{DISCUSSION}

Our descriptive analysis of four countries across the southern and the northern hemispheres provides compelling evidence of the potential association between the waves of the COVID-19 pandemic, the introduction of behavior-based COVID-19 mitigation measures, and a reduction in influenza transmission. There are two notable implications stemming from our 


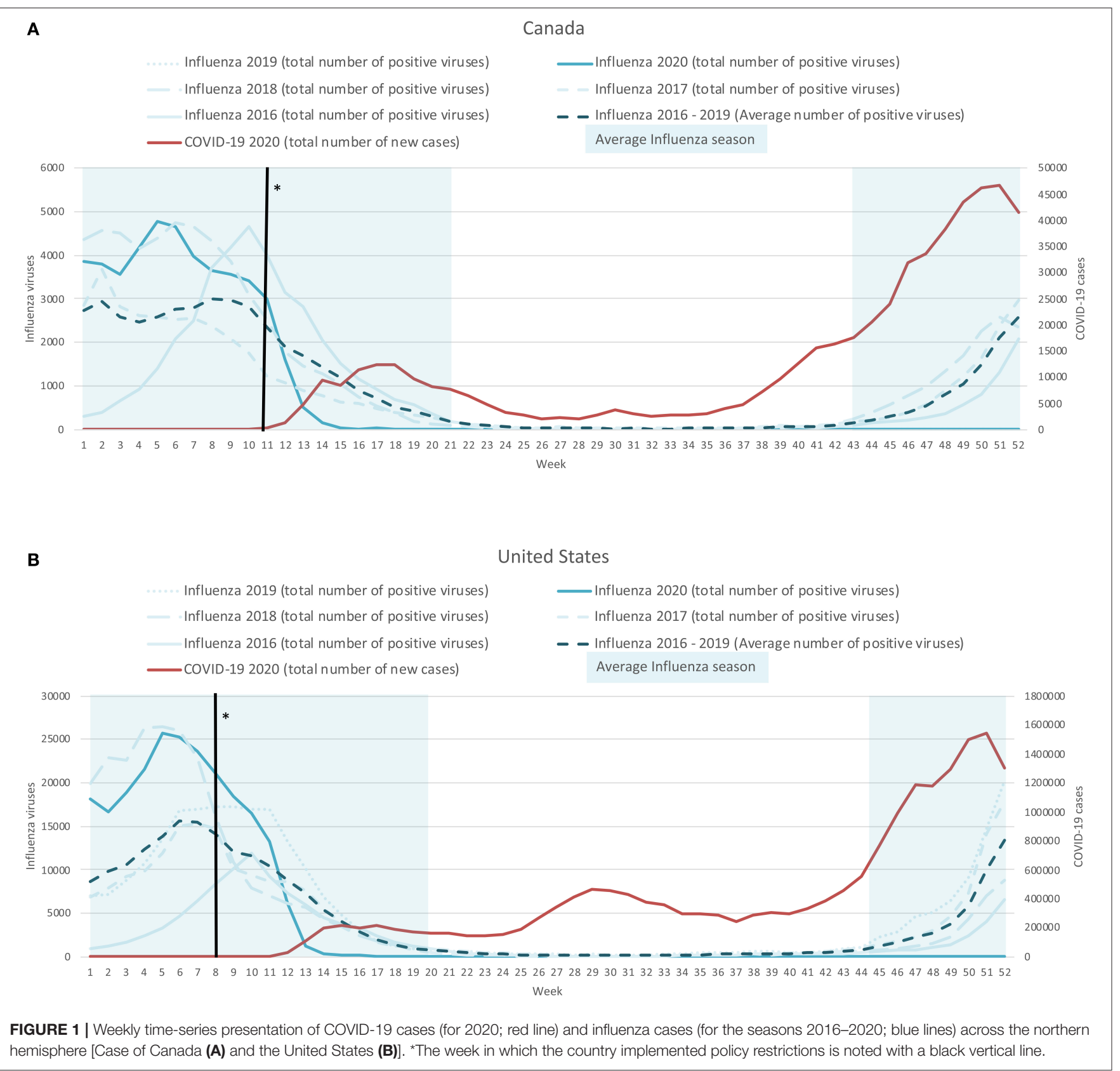

visual analysis, including: (1) the COVID-19 behavioral mitigation measures appear to be having an unintended positive consequence on influenza spread; and (2) the fact that there were still COVID-19 cases after the introduction of mitigation measures, and in the absence of community spread of influenza, suggests that these viruses are not equally transmissible.

With regards to the first point, even though our analyses are descriptive in nature, our hypothesis that the introduction of government measures, such as the adoption of widespread behavioral changes worldwide with respect to isolation, hygiene and social distancing in response to COVID-19 would have reduced the trends in annual influenza cases, remains plausible (12-14). We observed consistent reductions in influenza activity across the globe, which was most notable once governments had introduced their first measures. It is also worth highlighting that the four countries introduced their measures at different times in the year, yet their impact on the incidence of influenza cases was comparably rapid. Once the world has overcome the current COVID-19 pandemic crisis, consideration of the potential role of more rigorous and widespread implementation of the COVID-19 behavior-based prevention measures to curb the transmission of the influenza virus, and its global mortality burden (15), is needed.

To further emphasize the role of the behavior-based measures is the fact that most countries, including the United States and Canada, did not run a flu vaccine campaign for 2020, 


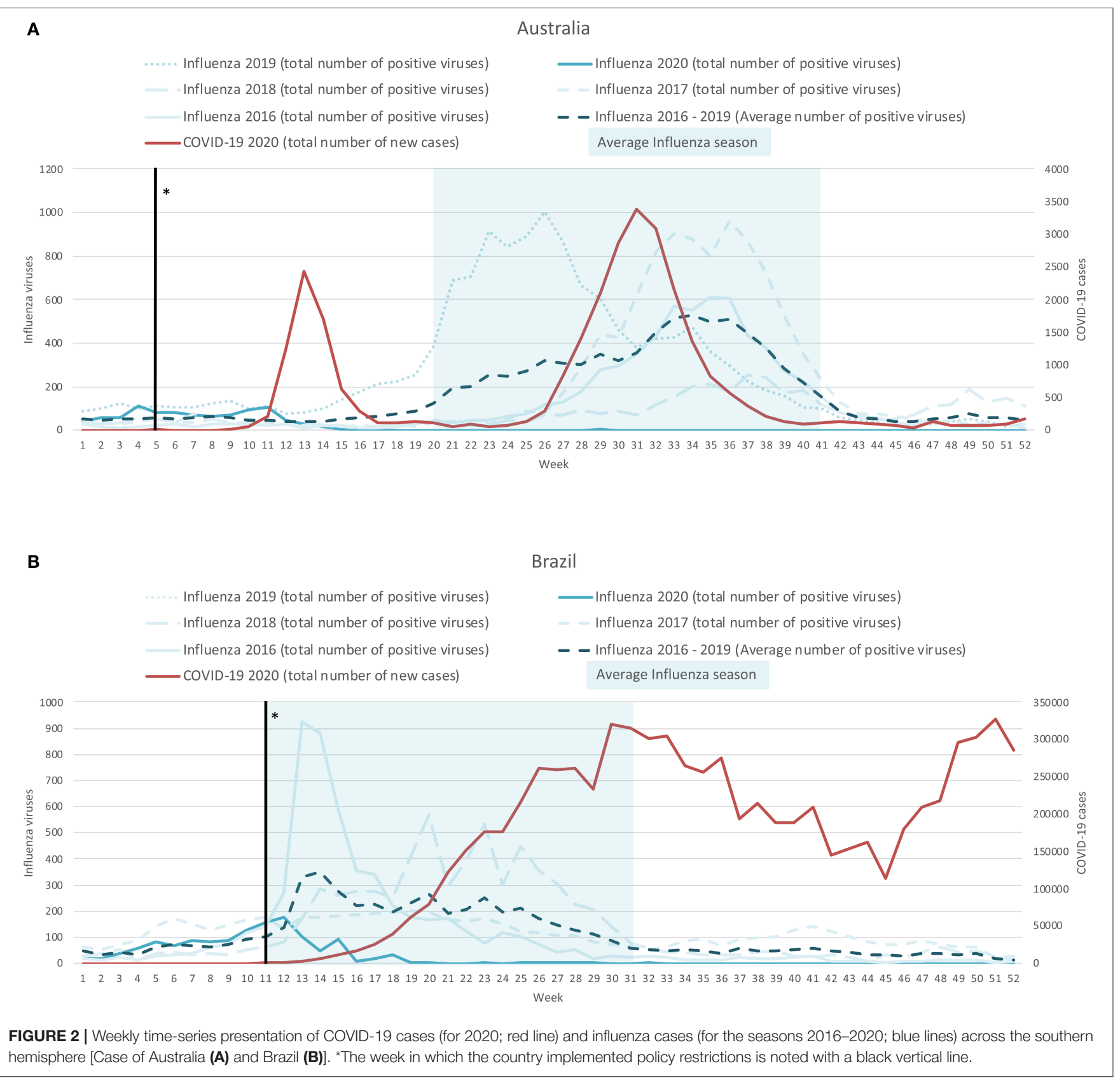

yet still managed to negate the usual incidence of influenza cases. This contrasts with Australia who ran an enhanced 2020 influenza vaccination campaign. When considering the impending distribution of the various COVID-19 vaccines, it might be possible for countries to drawn inspiration from this highly successful campaign, especially given the alarming increases in COVID-19 vaccine hesitancy (16). Elements of the Australian campaign included: continuous vaccination offer; aggressive public health messaging to inform the citizens, encourage vaccination and highlight the "it's never too late to get the vaccine" approach; increased advocacy for preventive behaviors; targeted messaging among at risk populations and healthcare workers; continuous surveillance and active monitoring (17). Importantly, it would seem that the development of public health policies and communication strategies aimed at increasing vaccine uptake might benefit from consultation with behavioral scientists, especially as the act of getting vaccinated is an important health behavior, whose insights have proven invaluable in the context of reducing COVID-19 transmission $(18,19)$.

Regarding our second implication, even though many of the parallels drawn between COVID-19 and influenza have already been discredited (20-26), it is often difficult to communicate this to the wider population. Our epidemiological mapping provides visually intuitive support to this difference which can help combat the misinformation that the impact of COVID-19 is 
no different to influenza. The current data clearly show how the two differ with respect to their infectiousness. The fact that there continues to be increases in COVID-19 cases with concomitant reductions in influenza spread around the globe highlights how viral the SARS-CoV-2 virus is.

\section{LIMITATIONS}

Our study should be interpreted in consideration of some limitations. Firstly, our methodological approach included a simple graphical visualization strategy of raw data, and so causality cannot strictly be inferred from this descriptive analysis. Extending this, there could be some case misclassification with some COVID-19 cases actually being influenza cases. However, it should be noted that the data that we used was generally based on actual testing of both COVID-19 and influenza rather than just symptom reporting. Another limitation involves the nature of the recent influenza surveillance data. Case declines observed might be due to decreased testing over the course of the pandemic, as well as limited capacity for reporting in certain countries. This might be especially pertinent to low and middle-income countries where there is a historical lack of reliable estimates for influenza surveillance data. However, in the four countries highlighted in this paper this possible limitation might only apply to Brazil.

\section{CONCLUSION}

Our report provides descriptive evidence that the behaviorbased COVID-19 mitigation measures are likely to be associated with an important reduction in the transmission and impact of influenza. In spite of this reduction in influenza, there was still community spread of COVID-19, highlighting that SARS-CoV2 is markedly more contagious compared to influenza. These graphs, together with the higher global mortality rates of SARSCoV-2 compared to influenza, provide clear evidence that the impact of COVID-19 is far greater than influenza. Finally, greater implementation of some of the key behavior-based COVID19 mitigation measures $(18,19,27)$ to reduce the mortality and burden of future influenza outbreaks should be considered by governments.

\section{REFERENCES}

1. Timeline of WHO's response to COVID-19. Available online at: https://www.who.int/emergencies/diseases/novel-coronavirus-2019/ interactive-timeline?gclid=CjwKCAjwh7H7BRBBEiwAPXjadvDvchuZJf1 1e 8oFXHcbPXqa1uHbFQqahfZDgIdJBVsAY1_xJV-Q8RoCbQUQAvD_BwE\#! (accessed October 30, 2020).

2. Center for Systems Science and Engineering (CSSE) at Johns Hopkins University. Coronavirus COVID-19 (2019-nCoV) Dashboard. Available online at: https://gisanddata.maps.arcgis.com/apps/opsdashboard/index.html\#/ bda7594740fd40299423467b48e9ecf6 (accessed October 30, 2020).

3. Haug N, Geyrhofer L, Londei A, Dervic E, Desvars-Larrive A, Loreto V, et al. Ranking the effectiveness of worldwide COVID-19 government interventions. Nat Hum Behav. (2020) 4:1303-12. doi: 10.1038/s41562-020-01009-0

4. Nussbaumer-Streit B, Mayr V, Dobrescu AI, Chapman A, Persad E, Klerings I, et al. Quarantine alone or in combination with other public health measures

\section{DATA AVAILABILITY STATEMENT}

The data presented in this study come from publically available online repositories. The names of the repository/repositories and accession links can be found at: FluNet (www.who.int/flunet), Global Influenza Surveillance and Response System (GISRS) (available at: https://apps.who.int/flumart/Default?ReportNo= 2); The Oxford COVID-19 Government Response Tracker (OxCGRT) (available at: https://github.com/OxCGRT/covidpolicy-tracker/tree/master/data); Center for Systems Science and Engineering at Johns Hopkins University. Coronavirus COVID19 (2019-nCoV) Dashboard (available at: https:/github.com/ CSSEGISandData/COVID-19).

\section{AUTHOR CONTRIBUTIONS}

JS: conception and design, data analysis, interpretation of data, drafting the article, article reviewing and critical revision. VB: conception and design, data acquisition and analysis, interpretation of data, drafting the article, article reviewing and critical revision. JB, JE, and KL: conception and design, interpretation of data, article reviewing and critical revision. SB: conception and design, interpretation of data, drafting the article, article reviewing and critical revision.

\section{FUNDING}

iCARE was supported by the Canadian Institutes of Health Research (CIHR: SMC-151518, PI: SB), Fonds de recherche du Québec - santé (FRQ-S: 251618, PI: SB; and 34757, PI: KL), and the Fonds de recherche du Québec - Société et culture (FRQSC: 2019-SE1-252541, PI:SB). Study sponsors had no role in the design of the database and data collection.

\section{ACKNOWLEDGMENTS}

The authors would like to acknowledge all the iCARE study staff and collaborators who have provided support for the broader COVID-19 related research that this is informing (www.icarestudy.com).

to control COVID-19: a rapid review. Cochrane Database Syst Rev. (2020) 4:CD013574. doi: 10.1002/14651858.CD013574.pub2

5. Burns J, Movsisyan A, Stratil JM, Coenen M, Emmert-Fees KMF, Geffert $\mathrm{K}$, et al. Travel-related control measures to contain the COVID-19 pandemic: a rapid review. Cochrane Database Syst Rev. (2020) 10:CD013717. doi: 10.1002/14651858.CD013717

6. Li Y, Reeves RM, Wang X, Bassat Q, Brooks WA, Cohen C, et al. Global patterns in monthly activity of influenza virus, respiratory syncytial virus, parainfluenza virus, and metapneumovirus: a systematic analysis. Lancet Glob Heal. (2019) 7:e103145. doi: 10.1016/S2214-109X(19) 30264-5

7. Facebook Removes Trump Post Falsely Saying Flu Is More Lethal Than Covid - CNN. Available online at: https://www.cnn.com/2020/10/06/tech/facebooktrump-covid-flu-false/index.html (accessed October 30, 2020).

8. Anti-Mask Protest in Montreal Draws Large Crowd, Propelled by U.S. Conspiracy Theories. CBC News. Available online at: https://www.cbc. 
ca/news/canada/montreal/anti-mask-protest-montreal-1.5722033 (accessed October 30, 2020).

9. WHO Flumart Outputs. Available at: https://apps.who.int/flumart/Default? ReportNo=2 (accessed October 30, 2020).

10. Dong E, Du H, Gardner L. An interactive web-based dashboard to track COVID-19 in real time. Lancet Infect Dis. (2020) 20:533-4. doi: 10.1016/S1473-3099(20)30120-1

11. Hale T, Webster S, Petherick A, Phillips TKB. Oxford covid-19 government response tracker. Blavatnik School of Government. Data use policy: Creative Commons Attribution CC BY standard (2020).

12. Carlson CJ, Gomez ACR, Bansal S, Ryan SJ. Misconceptions about weather and seasonality must not misguide COVID-19 response. Nat Commun. (2020) 11:4312. doi: 10.1038/s41467-020-18150-z

13. Baker RE, Park SW, Yang W, Vecchi GA, Jessica C, Grenfell BT. The impact of COVID-19 nonpharmaceutical interventions on the future dynamics of endemic infections. Proc Natl Acad Sci USA. (2020) 117:30547-53. doi: 10.1073/pnas.2013182117

14. Jones N. How COVID-19 is changing the cold and flu season. Nature. (2020) 588:388-90. doi: 10.1038/d41586-020-03519-3

15. Iuliano $\mathrm{AD}$, Roguski $\mathrm{KM}$, Chang $\mathrm{HH}$, Muscatello DJ, Palekar R, Tempia S, et al. Estimates of global seasonal influenza-associated respiratory mortality: a modelling study. Lancet. (2018) 391:1285-300. doi: 10.1016/S0140-6736(17)33293-2

16. Lazarus JV, Ratzan SC, Palayew A, Gostin LO, Larson HJ, Rabin K, et al. A global survey of potential acceptance of a COVID-19 vaccine. Nat Med. (2020) 27:225-8. doi: 10.1038/s41591-020-1124-9

17. Australian Government Department of Health. Seasonal Influenza Infection. CDNA National Guidelines for Public Health Units. Australian Government Department of Health and Ageing (2017). Available online at: https://www1. health.gov.au/internet/main/publishing.nsf/Content/cdna-song-influenza. htm (accessed October 30, 2020).

18. Betsch C. How behavioural science data helps mitigate the COVID-19 crisis. Nat Hum Behav. (2020) 4:438. doi: 10.1038/s41562-020-0866-1

19. Michie S, West R. Behavioural, environmental, social, and systems interventions against covid-19. BMJ. (2020) 370:m2982. doi: 10.1136/bmj.m2982

20. Petersen E, Koopmans M, Go U, Hamer DH, Petrosillo N, Castelli F, et al. Comparing SARS-CoV-2 with SARS-CoV and influenza pandemics. Lancet Infect Dis. (2020) 20:e238-44. doi: 10.1016/S1473-3099(20) 30484-9

21. Stowe J, Tessier E, Zhao H, Guy R, Muller-Pebody B, Zambon M, et al. Interactions between SARS-CoV-2 and Influenza and the impact of coinfection on disease severity: a test negative design. medRxiv. (2020) 2020.09.18.20189647. doi: 10.1101/2020.09.18.20189647
22. Van Damme W, Dahake R, Delamou A, Ingelbeen B, Wouters E, Vanham G, et al. The COVID-19 pandemic: diverse contexts; different epidemics - how and why? BMJ Glob Heal. (2020) 5:3098. doi: 10.1136/bmjgh-2020-003098

23. Zhou X, Ding G, Shu T, Fu S, Tong W, Tu X, et al. The outbreak of coronavirus disease 2019 interfered with influenza in Wuhan. Lancet. (2020). doi: 10.2139/ssrn.3555239

24. Zipfel C, Bansal S. Assessing the interactions between COVID-19 and influenza in the United States. medRxiv Prepr Serv Heal Sci. (2020) 2020.03.30.20047993. doi: 10.1101/2020.03.30.20047993

25. Petti S, Cowling BJ. Ecologic association between influenza and COVID19 mortality rates in European countries. Epidemiol Infect. (2020) 148:e209. doi: 10.1017/S0950268820002125

26. Pormohammad A, Ghorbani S, Khatami A, Razizadeh MH, Alborzi E, Zarei $\mathrm{M}$, et al. Comparison of influenza type $\mathrm{A}$ and $\mathrm{B}$ with COVID19: a global systematic review and meta-analysis on clinical, laboratory and radiographic findings. Rev Med Virol. (2020) 9:e2179. doi: 10.1002/rm v.2179

27. Bavel JJV, Baicker K, Boggio PS, Capraro V, Cichocka A, Cikara M, et al. Using social and behavioural science to support COVID-19 pandemic response. Nat Hum Behav. (2020) 4:460-471. doi: 10.1038/s41562-020-0 $884-\mathrm{z}$

Conflict of Interest: SB has received consultancy fees from Merck for the development of behavior change continuing education modules, speaker fees from Novartis and Janssen, and has served on advisory boards for Bayer, Sanofi, and Sojecci Inc none of which are related to the current article. KL has served on the advisory board for Schering-Plow, Takeda, AbbVie, Almirall, Janssen, GSK, Boehringer Ingelheim (BI), and Sojecci Inc, and received sponsorship for investigator-generated research grants from GlaxoSmithKline (GSK) and AbbVie, speaker fees from GSK, Astra-Zeneca, Astellas, Novartis, Takeda, AbbVie, Merck, Boehringer Ingelheim, Bayer, Pfizer and Air Liquide, and support for educational materials from Merck, none of which are related to the current article.

The remaining authors declare that the research was conducted in the absence of any commercial or financial relationships that could be construed as a potential conflict of interest.

Copyright (C) 2021 Stojanovic, Boucher, Boyle, Enticott, Lavoie and Bacon. This is an open-access article distributed under the terms of the Creative Commons Attribution License (CC BY). The use, distribution or reproduction in other forums is permitted, provided the original author(s) and the copyright owner(s) are credited and that the original publication in this journal is cited, in accordance with accepted academic practice. No use, distribution or reproduction is permitted which does not comply with these terms. 